\title{
Sign problem in two-color two-flavor QCD with quark and isospin chemical potentials
}

\author{
Kenji Fukushima*t \\ Yukawa Institute for Theoretical Physics, Kyoto University, Kyoto 606-8502, Japan \\ E-mail: fuku@yukawa.kyoto-u.ac.jp
}

We revisit two-color two-flavor Quantum Chromodynamics (QCD) which suffers from the sign problem of the Dirac determinant at finite baryon or quark density if a finite isospin chemical potential breaks two-flavor degeneracy. We discuss the eigenvalue distribution of the single-flavor Dirac operator to find the quartet structure, i.e. if $\lambda+m$ is an eigenvalue where $m$ is the current quark mass in a continuum theory, $\lambda^{*}+m,-\lambda+m$, and $-\lambda^{*}+m$ must be eigenvalues as well. As a result the product of these four eigenvalues makes a non-negative real number, unless $\lambda$ is real. The sign problem remains harmful for real $\lambda$ because not a quartet but a pair of $\lambda+m$ and $-\lambda+m$ appears then and its product is not necessarily non-negative. In such a case, however, the sign problem has a different nature from the ordinary one in three-color QCD at finite density. We can rather identify it as the sign problem in the parity broken (or Aoki) phase inherent in the Wilson fermion formalism.

The XXV International Symposium on Lattice Field Theory

July 30-4 August 2007

Regensburg, Germany

* Speaker.

${ }^{\dagger}$ Moved from RIKEN BNL Research Center on September 1st. 


\section{Introduction}

It is a longstanding problem to uncover the phase structure of dense nuclear and quark matter in the low temperature and high baryon density region which is quite complicated. From the academic point of view, our curiosity urges us to imagine what an extreme state of cold quark matter at asymptotic high density is like. In fact, at density far larger than the strange quark mass $M_{S}$ but still smaller than the charm, bottom, and top quark masses, there has been established a consensus that quark matter takes on color superconductivity in a color-flavor locked (CFL) manner. Then, what comes next as the density goes down? This is an important question because, if quark matter appears in neutron star cores, its state would be strongly affected by the "pressure" induced by $M_{s}$ which tends to tear the Cooper pair apart.

This "pressure" makes the phase structure of dense nuclear and quark matter quite complicated especially in the intermediate density region where the density is at most up to $10 \rho_{0}$ with $\rho_{0}$ being the normal nuclear density. So far, many candidates for the ground state at intermediate density have been proposed, among which a scenario attracting much theoretical interest in recent years is that the Larkin-Ovchinnikov-Fulde-Ferrell (LOFF) [1] state could take over the gapless region in which the "pressure" exceeds the superconducting pairing energy. In the LOFF state each Cooper pair carries a non-vanishing total momentum $2 \vec{q}$ which breaks translational and rotational symmetries spontaneously. Consequently we can naturally anticipate an inhomogeneous color superconductor, or specifically, a crystalline color superconductor [2]. This theoretical possibility is extremely interesting; the pairing gap takes a crystal structure in a superconducting fluid.

For the moment it is an unsolved problem to clarify the energetically most favored crystal structure in the LOFF state. We shall here revisit the LOFF phase in two-color two-flavor QCD in the presence of both the quark chemical potential $\mu_{q}$ and the isospin chemical potential $\mu_{I}$. It was pointed out in Ref. [3] that the interval of $\mu_{I}$ in which the plane-wave LOFF state is favored exists in such a system at least at weak coupling. Two-color QCD is known to be free from the sign problem of the Dirac determinant at finite density [4], and so, it would be great if one can look into the crystal structure of the two-color LOFF state by means of the Monte-Carlo simulation, which could provide us with useful hints to attack three-color QCD problems. It is, however, impossible to perform the simulation directly to reveal the crystal structure, as discussed in Ref. [3], because two (or an even number of) degenerate quark flavors are required in order that the sign problem is completely absent from two-color QCD. Since the isospin chemical potential is introduced to exert a "pressure" between different quark flavors, even two-color QCD would suffer from the sign problem with $\mu_{q} \neq 0$ and $\mu_{I} \neq 0$.

We shall closely examine how the sign problem could emerge in two-color single-flavor QCD in what follows. [It is enough to focus on the single-flavor case because the Dirac determinant with multiple flavors is just a product of the determinant for respective flavor sectors.] We will here not resolve but classify the sign problem; we will find that the sign problem in two-color single-flavor QCD is similar to the sign problem in the parity broken or the Aoki phase [5]. In a sense, we may say that the superfluid phase in two-color QCD could be roughly regarded as the Aoki phase in which the pion condensation spontaneously occurs, though we have to distinguish the baryonic pion condensate from the ordinary mesonic pion condensate. Here, I would like to refer to an interesting related work by Hands et al. [6]. 
In the final part of this article, we will briefly mention on the likely phase diagram of twocolor two-flavor QCD with non-zero $\mu_{q}$ and $\mu_{I}$. We will present a model prediction [7] for the phase boundary in the parameter region spanned by $\mu_{q}$ and $\mu_{I}$ and will locate the LOFF-favored window based on the mean-field calculation.

\section{Sign Problem and Parity Broken Phase}

We shall here summarize the sign problem of the Dirac determinant and discuss that two-color QCD nearly escapes from it for any number of quark flavors except for the possible parity broken phase. The single-flavor Dirac operator in question reads

$$
\mathscr{M}\left(\mu_{q}\right)=\gamma_{\mu} D^{\mu}+\gamma_{4} \mu_{q}+m
$$

in continuum Euclidean space, where $D^{\mu}$ denotes the covariant derivative containing gauge fields. In the presence of the isospin chemical potential $\mu_{I}$ with $u$ and $d$ flavors, the Dirac operator is then a direct sum of two flavor sectors: $\mathscr{M}\left(\mu_{u}\right) \oplus \mathscr{M}\left(\mu_{d}\right)$. We shall thus focus on the single-flavor $\mathscr{M}\left(\mu_{q}\right)$ to discuss the sign problem, since $\operatorname{det} \mathscr{M}\left(\mu_{q}\right) \geq 0$ is sufficient to claim $\operatorname{det}\left[\mathscr{M}\left(\mu_{u}\right) \oplus \mathscr{M}\left(\mu_{d}\right)\right]=$ $\operatorname{det} \mathscr{M}\left(\mu_{u}\right) \cdot \operatorname{det} \mathscr{M}\left(\mu_{d}\right) \geq 0$.

First of all, let us confirm that the zero-density continuum Dirac determinant is certainly nonnegative and real. Because the Euclidean gamma matrices $\gamma_{\mu}$ 's are hermitian by convention, the eigenvalue $\lambda_{n}$ of anti-hermitian $\gamma_{\mu} D^{\mu}$, i.e.,

$$
\gamma_{\mu} D^{\mu} \psi_{n}=\lambda_{n} \psi_{n}
$$

is pure imaginary. The eigenstate $\gamma_{5} \psi_{n}$ has an eigenvalue $-\lambda_{n}$, which equals $\lambda_{n}^{*}$. The Dirac determinant at zero density is, therefore, a product of all the paired eigenvalues $\lambda_{n}+m$ and $\lambda_{n}^{*}+m$, which is non-negative because $\left|\lambda_{n}+m\right|^{2} \geq 0$. In this case, the eigenvalue distribution is only on the line $\operatorname{Re} \lambda=m$, which is qualitatively illustrated in Fig. 1 (a).

When $\mu_{q}$ is nonzero, $\gamma_{4} \mu_{q}$ is hermitian and thus the eigenvalue $\lambda_{n}$ defined by

$$
\left(\gamma_{\mu} D^{\mu}+\gamma_{4} \mu_{q}\right) \psi_{n}=\lambda_{n} \psi_{n}
$$

is no longer pure imaginary but complex. Here again, $\gamma_{5} \psi_{n}$ has an eigenvalue $-\lambda_{n}$, but it is different from $\lambda_{n}^{*}$ in this case. Therefore, the Dirac determinant, given by $\prod_{n}\left(\lambda_{n}+m\right)\left(-\lambda_{n}+m\right)$, is not necessarily non-negative, and when it is negative for some gauge configurations, the sign problem occurs. If we sketch the eigenvalue distribution corresponding to such a situation, it should be tilted and spreading over the complex plane like Fig. 1(c). Here, so far, we have seen what is going on in general cases other than two-color QCD. Let us next turn to the case in two-color QCD.

Two-color QCD is unique in the sense that it has extra symmetry so that one can find another eigenstate $\sigma_{2} C^{-1} \gamma_{5} \psi_{n}^{*}$ with an eigenvalue $\lambda_{n}^{*}$, where $\sigma_{2}$ is the second Pauli matrix in color space and $C$ represents the charge conjugation. Obviously, one more eigenstate multiplied by $\gamma_{5}$ has an eigenvalue $-\lambda_{n}^{*}$. Consequently, the eigenvalues of the Dirac operator always constitute a quartet; $\lambda_{n}+m,-\lambda_{n}+m, \lambda_{n}^{*}+m$, and $-\lambda_{n}^{*}+m$, leading to the non-negative Dirac determinant through the relation $\left|\lambda_{n}+m\right|^{2} \cdot\left|-\lambda_{n}+m\right|^{2} \geq 0$. This argument holds as long as $\lambda_{n}$ is complex. If $\lambda_{n}$ happens to be real, however, there appear only $\lambda_{n}+m$ and $-\lambda_{n}+m$ instead of a quartet. The Dirac 
(a)

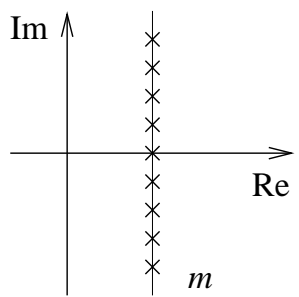

(b)

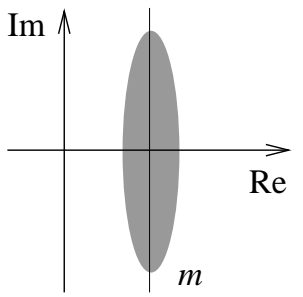

(c)

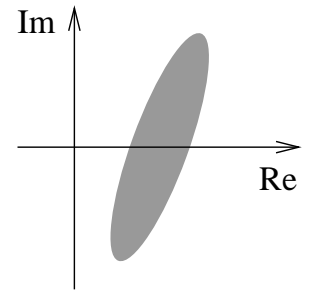

(d)

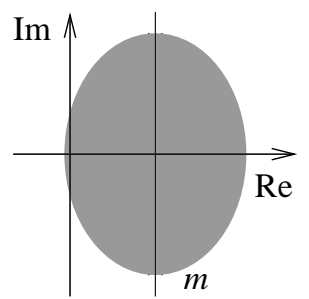

Figure 1: Qualitative sketches for the eigenvalue distribution of the Dirac operator (a) at zero density without the Wilson term, (b) at zero density with the Wilson term or at finite density in two-color QCD, (c) at finite density in QCD, and (d) in the Aoki phase at zero density in QCD or at zero/finite density in two-color QCD.

determinant becomes negative for $-\lambda_{n}+m<0$, while the determinant is real and the squared quantity is always non-negative. Thus, this is the reason we need two (or an even number of) quark flavors to guarantee the non-negative Dirac determinant [8].

Then, one might have thought that the sign problem remains after all and the above-mentioned clarification enables us to make no progress at all. What we would emphasize here is, however, that the sign problem in two-color QCD has totally different characteristics from the ordinary sign problem sketched in Fig. 1 (c). To see it, let us take one farther step to come close to the lattice formulation.

We could have put all our discussions below on the lattice but we will not do so. It is because the mass $m$ has to be replaced by the hopping parameter $\kappa$ in the lattice language and it is not transparent to make an intuitively comprehensible comparison to the situation in the continuum theory we have understood so far. Hence, instead of mathematically rigorous expressions in the lattice formulation, we shall stick to using a more familiar language in the continuum theory. Actually, the essential point in our discussions is only that the Dirac operator must have an extra term to eliminate the doublers in the Wilson fermion formalism. Of course, such a term, which is usually called the Wilson term, should be zero in the continuum limit, but if we keep the Wilson term in the continuum language, the Dirac operator takes a form of

$$
\mathscr{M}\left(\mu_{q}\right)=\gamma_{\mu} D^{\mu}+\gamma_{4} \mu_{q}+m+r D_{\mu} D^{\mu}
$$

with the Wilson coefficient $r$ which, in fact, goes to zero in the continuum limit. It is crucial to note that the operator $D_{\mu} D^{\mu}$ is hermitian, so that the eigenvalue $\lambda_{n}$ defined by

$$
\left(\gamma_{\mu} D^{\mu}+r D_{\mu} D^{\mu}\right) \psi_{n}=\lambda_{n} \psi_{n}
$$

is generally complex. How far the eigenvalue distribution can be away from the vertical line at $\operatorname{Re} \lambda=m$ depends on $r$. As long as $r$ stays small as compared with $m$, the eigenvalue distribution at $\mu_{q}=0$ is like Fig. 1 (b). Still, the Dirac determinant is non-negative real, as it should be. It is because of the quartet structure of the eigenvalues, $\lambda_{n}+m,-\lambda_{n}+m, \lambda_{n}^{*}+m$, and $-\lambda_{n}^{*}+$ $m$ [9]. This might sound confusing, but this quartet structure has nothing to do with the twocolor specialty. To avoid confusion, we should reiterate what we are addressing here. The system of our present interest is QCD itself at zero density $m u_{q}=0$ with non-zero Wilson coefficient $r \neq 0$. Strictly speaking, as the simulation approaches the weak-coupling or continuum limit, the eigenvalue distribution has to produce blank spots along the real axis [5, 9], but such details are irrelevant to our discussions. 
In the presence of finite baryon or quark chemical potential, the eigenvalue distribution is tilted with not a quartet but a pair of eigenvalues $\lambda_{n}+m$ and $-\lambda_{n}+m$ as shown in Fig. 1(c). Under such circumstances the Wilson term makes no qualitative difference for the sign problem occurring at finite density.

As a matter of fact, the Dirac determinant at zero density is not always safe from the sign problem once the Wilson term is added in the Lagrangian. One can easily imagine from Fig. 1 (b) what would transpire in the eigenvalue distribution if one goes on increasing $r$. The distribution would become wider along the real axis and eventually the edge would touch the origin (see Fig. 1 (d)). Then, the eigenvalues enter the negative region, $\operatorname{Re} \lambda_{n}<0$. If $\lambda_{n}$ is not real, nevertheless, the product of $\lambda_{n}+m$ and $\lambda_{n}^{*}+m$ still makes a non-negative real number. However, the eigenvalue distribution generally contains real $\lambda_{n}$ that does not have a partner $\lambda_{n}^{*}$ but sits on the real axis alone. Therefore, when such real $\lambda_{n}$ is smaller than $-m$, the sign problem occurs even at zero density! This kind of the sign problem has been well-known as realization of the parity broken or Aoki phase since seminal works by Aoki [5] more than two decades ago.

The physical interpretation for Fig. 1(d) is as follows. From the Banks-Casher relation in the pion channel, the non-zero eigenvalue density in the vicinity of the origin leads to a finite value of the pion condensate $\left\langle\bar{q} \gamma_{5} q\right\rangle$ that breaks parity and is usually vanishing in the case like Fig. 1 (a). The spontaneous generation of $\left\langle\bar{q} \gamma_{5} q\right\rangle \neq 0$ happens as the second-order phase transition with varying $m$ and $r$ and the correlation length between pion operators develops infinite at the critical point. This means that the system has the massless pion then. The chiral limit is, therefore, not the limit of $m \rightarrow 0$ but defined along the phase boundary $r=r_{c}(m)$ on which the pion is massless.

By now, the reader might have already noticed that what is happening in the parity broken phase is completely parallel to the sign problem in two-color QCD at finite density. Owing to extra symmetry peculiar to two-color QCD, the quartet structure in the eigenvalue distribution is maintained even in the presence of non-zero density. As a result the eigenvalue distribution in twocolor QCD at finite density is like either Fig. 1(b) or Fig. 1(d) rather than Fig. 1(c) depending on $\mu_{q}, m$, and $r$. Actually one cannot tell the sign problem in two-color QCD from the sign problem in the parity broken phase in principle. It implies that one should, in turn, distinguish the sign problem in two-color QCD from the sign problem in finite-density QCD.

It is a theoretically intriguing question of great significance to make clear the phase structure of the parity broken phase in two-color QCD at finite density. The numerical simulation is feasible because the Dirac determinant keeps being non-negative real until the parity broken phase occurs, so that the phase boundary is accessible where the "chiral limit" is achieved. It would be non-trivial whether this chiral limit on the phase boundary should correspond to the critical point associated with pion superfluidity or not. In the chiral limit the mesonic pion is massless, while on the onset of superfluidity the baryonic pion is massless. Since the particle-antiparticle symmetry (Pauli-Gürsey symmetry) is explicitly broken by $\mu_{q} \neq 0$, it is far from obvious that two criticality should be achieved at the same time. My conjecture is that the "chiral limit" in fact turns out to be a critical point for superfluidity, which would be able to be tested by the hopping parameter expansion at strong coupling along a similar line to Ref. [10], or in the direct numerical simulation. 

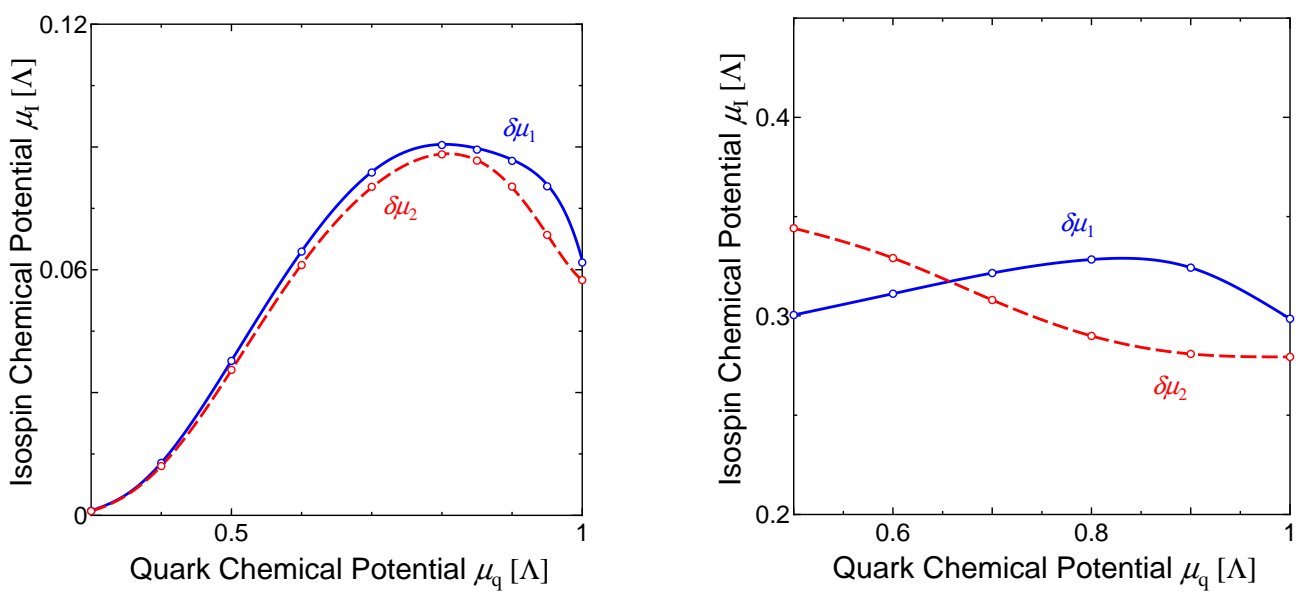

Figure 2: Mean-field predictions for the phase structure of two-color two-flavor QCD in the $\mu_{q}$ and $\mu_{I}$ plane with only the single plane-wave LOFF state taken into account. The LOFF state occurs in the region $\delta \mu_{2}<\mu_{I}<\delta \mu_{1}$. The left figure is for weak coupling with the superfluid gap $\Delta\left(\mu_{q}=0.5 \Lambda, \mu_{I}=0\right)$ chosen to be $0.05 \Lambda$, and the right for stronger coupling with $\Delta_{0}\left(\mu_{q}=0.5 \Lambda, \mu_{I}=0\right)$ chosen to be $0.5 \Lambda$. In both cases a uniform superfluid is favored for $\mu_{I}<\delta \mu_{1}$ or $\mu_{I}<\delta \mu_{2}$.

\section{Phase Diagram}

Finally, we shall make an overview for physical implications we can expect if we somehow manage to resolve the sign problem in two-color single-flavor QCD with $\mu_{q} \neq 0$ or in two-color two-flavor QCD with $\mu_{q} \neq 0$ and $\mu_{I} \neq 0$. As already mentioned in Introduction, one of the most interesting applications is the analysis on the LOFF state on the lattice.

In Ref. [7], Kei Iida and the present author have given mean-field predictions for the phase diagram in the $\mu_{q}$ and $\mu_{I}$ plane. There, the coupling strength is specified by the magnitude of the superfluid gap or the diquark condensate $\Delta_{0}$ at a certain density (chosen arbitrarily to be $\mu_{q}=0.5 \Lambda$ ) with $\mu_{I}=0$, where $\Lambda$ is the ultraviolet cutoff in momentum space that corresponds to the lattice spacing in configuration space.

In the weak-coupling case $\left(\Delta_{0}=0.05 \Lambda\right)$ we found the interval of $\mu_{I}$ in which the single planewave LOFF state has a lower energy than a uniform superfluid. As long as $\mu_{q}$ is not so large as the saturation effect is evident, the LOFF-favored window is $0.71 \Delta<\mu_{I}<0.75 \Delta$ that is well consistent with the known perturbative results [1]. In the stronger-coupling case $\left(\Delta_{0}=0.5 \Lambda\right)$ that is more relevant to the possible lattice-simulation environment, the LOFF window does not appear until $\mu_{q}$ is larger than about $0.65 \Lambda$. In the higher density region the LOFF-favored window opens with enhancement by the saturation effect. Here, we should emphasize that the windows indicated in Fig. 2 are sufficient conditions for some state other than a uniform superfluid to be the ground state. The single plane-wave is the simplest and presumably worst ansatz to describe the LOFF state; the realistic crystalline phase must have a wider window that embraces the LOFF-favored window under the single plane-wave ansatz. In view of Fig. 2 I think that we have a good chance to observe the LOFF state on the lattice.

To do so, needless to say, we cannot walk around the problem to understand how the sign 
problem in the parity broken phase is involved in superfluidity. Usually, one dismisses the parity broken phase as an unphysical state, but in the two-color QCD system, it could be a physical state. Hence, in this sense, the possible relation between superfluidity and the parity broken phase is a profound problem useful for us to acquire a deeper insight into the sign problem from the physical point of view.

\section{Acknowledgment}

The author thanks Kei Iida, Atsushi Nakamura, and Chiho Nonaka for a stimulating collaboration whose name is the MOMIJI collaboration. MOMIJI is a Japanese word meaning MAPLE that is an abbreviation of "MAny-body Physics on the Lattice is Excellent!" The author learned a lot through the collaboration. Of course, if any, only the present author is responsible for any criticism.

\section{References}

[1] A. I. Larkin and Yu. N. Ovchinnikov, Sov. Phys. JETP 20 (1965) 762; P. Fulde and R. A. Ferrell, Phys. Rev. 135 (1964) A550.

[2] M. G. Alford, J. A. Bowers and K. Rajagopal, Phys. Rev. D 63 (2001) 074016.

[3] K. Splittorff, D. T. Son and M. A. Stephanov, Phys. Rev. D 64 (2001) 016003.

[4] A. Nakamura, Phys. Lett. B 149 (1984) 391.

[5] S. Aoki, Phys. Rev. D 30 (1984) 2653; S. Aoki and A. Gocksch, Phys. Rev. D 45 (1992) 3845.

[6] S. Hands, I. Montvay, L. Scorzato and J. Skullerud, Eur. Phys. J. C 22 (2001) 451.

[7] K. Fukushima and K. Iida, Phys. Rev. D 76 (2007) 054004.

[8] M. G. Alford, A. Kapustin and F. Wilczek, Phys. Rev. D 59 (1999) 054502.

[9] R. Setoodeh, C. T. H. Davies and I. M. Barbour, Phys. Lett. B 213 (1988) 195.

[10] Y. Nishida, K. Fukushima and T. Hatsuda, Phys. Rept. 398 (2004) 281. 